\title{
Torsional path integral Monte Carlo method for the quantum simulation of large molecules
}

\author{
Thomas F. Miller III and David C. Clarya) \\ Department of Chemistry, University College London, 20 Gordon Street, London WC1H OAJ, \\ United Kingdom
}

(Received 24 October 2001; accepted 12 February 2002)

\begin{abstract}
A molecular application is introduced for calculating quantum statistical mechanical expectation values of large molecules at nonzero temperatures. The Torsional Path Integral Monte Carlo (TPIMC) technique applies an uncoupled winding number formalism to the torsional degrees of freedom in molecular systems. The internal energy of the molecules ethane, $n$-butane, $n$-octane, and enkephalin are calculated at standard temperature using the TPIMC technique and compared to the expectation values obtained using the harmonic oscillator approximation and a variational technique. All studied molecules exhibited significant quantum mechanical contributions to their internal energy expectation values according to the TPIMC technique. The harmonic oscillator approximation approach to calculating the internal energy performs well for the molecules presented in this study but is limited by its neglect of both anharmonicity effects and the potential coupling of intramolecular torsions. (C) 2002 American Institute of Physics. [DOI: 10.1063/1.1467342]
\end{abstract}

\section{INTRODUCTION}

A number of recent publications have suggested the utility of Path Integral Monte Carlo (PIMC) techniques for exploring the quantum mechanical properties of molecular systems. ${ }^{1-21}$ Marx and co-workers have been particularly successful in describing systems of molecular rotors in the condensed state and on surfaces. ${ }^{14,15}$ Also, Martoňák and coworkers have performed a comparison of the PIMC technique and a quasiharmonic technique in analyzing the bulk properties of crystalline polyethylene. ${ }^{20,21}$ However, few successful reports have been published on applications of PIMC techniques to large molecules using realistic potentials. ${ }^{11-13}$ Lee and Berne employ path integrals in the formulation of a quantum thermal annealing technique for locating the global minimum of proteins, ${ }^{11,12}$ and Dewey derives a statistical mechanical treatment of large biomolecules utilizing the sequence information as an internal coordinate. ${ }^{13}$ The current authors attempt to more fully realize the potential of the PIMC technique to calculate useful quantum statistical mechanical properties for large molecules at nonzero temperatures using a realistic structural model.

A recent diffusion Monte Carlo (DMC) study of proteins suggests that quantum dynamical simulations of large molecules may be possible. ${ }^{22}$ Arguing on the basis that torsional degrees of freedom predominate the most critical features of intramolecular motion in large systems such as proteins, the DMC simulation is restricted to only those torsional modes. Similar employment of a PIMC technique will likewise benefit from the restricted coordinate space while, unlike the DMC method, enabling investigation of molecular properties at nonzero temperatures.

In this study, we employ a torsional PIMC (TPIMC) technique formulated from an uncoupled winding number

\footnotetext{
a) Author to whom correspondence should be addressed; electronic mail: d.c.clary@ucl.ac.uk
}

representation of rotational path integrals. ${ }^{23}$ The technique is used to calculate the internal energy at standard temperature of an assortment of molecules ranging from 8 to 75 atoms (1-33 torsions). The largest system explored is the peptide enkephalin. Section II presents the torsional path integral formalism for a general system of $N$ torsions and reviews the winding number and uncoupled winding number formulations. ${ }^{14,23}$ Section III describes the details of the calculations and molecular systems presented in the study, and Sec. IV presents and discusses results. The conclusions in Sec. V discuss the potential of this technique for treating the quantum dynamics of macromolecules such as proteins.

\section{THEORY}

The PIMC method combines the distinct techniques of path integral theory and Monte Carlo theory. ${ }^{1-8}$ The path integral formalism descends directly from Feynman's manipulations of the canonical density matrix. ${ }^{24-26}$ Standard Monte Carlo techniques are utilized to solve the path integral and calculate properties of the system. ${ }^{27-30}$

\section{A. Derivation of torsional path integrals}

The path integral technique is remarkable for its suitability to problems of quantum statistical mechanics. ${ }^{24,25}$ Our derivation of path integrals for a system of $N$ torsions proceeds largely by analogy to classical statistical mechanics. We begin with the familiar canonical partition function

$$
\begin{aligned}
Q(\beta) & =\prod_{j=1}^{N}\left[\int_{0}^{2 \pi} d \theta_{j}\right]\left\langle\vec{\theta}\left|e^{-\beta H}\right| \vec{\theta}\right\rangle \\
& =\prod_{j=1}^{N}\left[\int_{0}^{2 \pi} d \theta_{j}\right] \rho(\vec{\theta}, \vec{\theta} ; \beta),
\end{aligned}
$$

where $\beta=1 / k T$. The term $\vec{\theta}$ represent the vector of the $N$ torsion positions. Equation (1) introduces the density matrix 
element $\rho(\vec{\theta}, \vec{\theta} ; \beta)$ which is a position matrix element of the Boltzmann-like operator $e^{-\beta H}$. It is clear that $Q(\beta)$ is the position trace of $\rho$ and is a function of temperature only via the variable $\beta$. This simple functional dependence of the partition function on temperature is a feature that is preserved throughout the development of path integrals.

The completeness of the quantum states yields the following useful property of the density matrix element:

$\rho\left(\vec{\theta}, \vec{\theta}^{\prime} ; \beta\right)$

$$
=\prod_{j=1}^{N}\left[\int_{0}^{2 \pi} d \theta_{j}^{\prime \prime}\right] \rho\left(\vec{\theta}, \vec{\theta}^{\prime \prime} ; \beta / 2\right) \rho\left(\vec{\theta}^{\prime \prime}, \vec{\theta}^{\prime} ; \beta / 2\right) .
$$

By inductively applying Eq. (2), we arrive at a discretized form of the canonical partition function

$$
Q(\beta)=\prod_{j=1}^{N} \prod_{t=1}^{P}\left[\int_{0}^{2 \pi} d \theta_{j, t}\right] \prod_{t=1}^{P} \rho\left(\vec{\theta}_{t}, \vec{\theta}_{t+1} ; \beta / P\right),
$$

where $\vec{\theta}_{1}=\vec{\theta}_{P+1}$. The vector $\vec{\theta}_{t}$ of length $N$ is composed of the $\theta_{j, t}$ angles corresponding to a particular discretization slice $t$. Equation (3), while preserving mathematical exactness, allows the canonical partition function to be written in terms of the product of $P$ high temperature density matrix elements of the form $\rho\left(\vec{\theta}_{t}, \vec{\theta}_{t+1} ; \beta / P\right)$. The strategy of the path integral theory is to accurately approximate these high temperature density matrix elements and subsequently evaluate the quantum partition function.

Chandler and Wolynes described the discretization of the canonical partition function as a classical isomorphism in which, conceptually, each classical particle is replaced by a ring of particles facilitating quantum behavior. ${ }^{2}$ These particles in the ring are known as Trotter beads. The integer $P$ is the number of Trotter beads.

The Trotter product formula applied to the density matrix operator $\hat{\rho}(\beta / P)$ yields, assuming $H=T+V:^{31-33}$

$$
\begin{aligned}
\hat{\rho}(\beta / P)= & e^{-\beta V / 2 P} e^{-\beta T / P} e^{-\beta V / 2 P} \\
& +\mathcal{O}\left[(\beta / P)^{3}\right] .
\end{aligned}
$$

Moreover, the Trotter theorem states that ${ }^{34}$

$$
\hat{\rho}(\beta / P)=\lim _{P \rightarrow \infty}\left[e^{-\beta V / 2 P} e^{-\beta T / P} e^{-\beta V / 2 P}\right]^{P} .
$$

Taking the position matrix element of $\hat{\rho}$ in Eq. (5) and substituting it into Eq. (3) leads to the result

$$
Q(\beta)=\lim _{P \rightarrow \infty} \prod_{j=1}^{N} \prod_{t=1}^{P}\left[\int_{0}^{2 \pi} d \theta_{j, t}\right] \prod_{t=1}^{P} \widetilde{\rho}\left(\vec{\theta}_{t}, \vec{\theta}_{t+1} ; \beta / P\right),
$$

where $\vec{\theta}_{1}=\vec{\theta}_{P+1}$ and

$$
\begin{aligned}
\widetilde{\rho}\left(\vec{\theta}_{t}, \vec{\theta}_{t+1} ; \beta / P\right)= & \left\langle\vec{\theta}_{t}\left|e^{-\beta T / P}\right| \vec{\theta}_{t+1}\right\rangle\left\{\exp -\frac{\beta}{2 P}\left(V\left(\vec{\theta}_{t}\right)\right.\right. \\
& \left.\left.+V\left(\vec{\theta}_{t+1}\right)\right)\right\} .
\end{aligned}
$$

Note from the error term in Eq. (4) that approximation of the matrix element $\rho\left(\vec{\theta}_{t}, \vec{\theta}_{t+1} ; \beta / P\right)$ with $\widetilde{\rho}\left(\vec{\theta}_{t}, \vec{\theta}_{t+1} ; \beta / P\right)$ improves with higher temperature or a larger number of Trotter beads.
For a system of $N$ torsions, the kinetic energy operator $T$ is taken as

$$
T=\sum_{j=1}^{N} \frac{-\hbar^{2}}{2 I_{j}} \frac{\partial^{2}}{\partial \theta_{j}^{2}},
$$

where $I_{j}$ is the moment of inertia of the $j$ th torsion. Torsional coupling terms have been neglected in Eq. (8), because for molecular systems, the kinetic coupling terms are far less significant than the terms arising from potential coupling which will be retained throughout this path integral development. ${ }^{35}$ By inserting an identity operator composed of free-rotor wave functions and utilizing a Poisson summation formula, we obtain the following relationship from Eq. $(7)::^{36,37}$

$$
\begin{aligned}
\widetilde{\rho}\left(\vec{\theta}_{t}, \vec{\theta}_{t+1} ; \beta / P\right) \\
=\prod_{j=1}^{N}\left[\left(\frac{I_{j} P}{2 \pi \hbar^{2} \beta}\right)^{1 / 2}\right. \\
\left.\quad \times \sum_{n_{j, t=-\infty}}^{\infty} \exp \left\{-\beta\left[\frac{I_{j} P}{2 \hbar^{2} \beta^{2}}\left(\theta_{j, t}-\theta_{j, t+1}+2 \pi n_{j, t}\right)^{2}\right]\right\}\right] \\
\quad \times\left\{\exp -\frac{\beta}{2 P}\left(V\left(\vec{\theta}_{t}\right)+V\left(\vec{\theta}_{t+1}\right)\right)\right\} .
\end{aligned}
$$

The combination of Eqs. (6) and (9) yields an exact form for the discretized canonical partition function of a system of $N$ torsions, ${ }^{14}$

$$
\begin{aligned}
Q(\beta)= & \lim _{P \rightarrow \infty}\left(\frac{P}{2 \pi \beta \hbar^{2}}\right)^{N P / 2} I^{1 / 2} \\
& \times \prod_{t=1}^{P} \prod_{j=1}^{N}\left[\sum_{n_{j, t}=-\infty}^{\infty} \int_{0}^{2 \pi} d \theta_{j, t}\right] e^{-\beta\left(V_{n}^{\text {int }}+V^{\text {ext }}\right)},
\end{aligned}
$$

where $P$ is the number of Trotter beads for the system, $\vec{\theta}_{1}$ $=\vec{\theta}_{P+1}, I=\prod_{k=1}^{N} I_{k}$, and with

$$
V_{n}^{\mathrm{int}}=\frac{P}{2 \beta^{2} \hbar^{2}} \sum_{t=1}^{P} \sum_{j=1}^{N} I_{j}\left(\theta_{j, t}-\theta_{j, t+1}+2 \pi n_{j, t}\right)^{2}
$$

and

$$
V^{\mathrm{ext}}=\frac{1}{P} \sum_{t=1}^{P} V\left(\vec{\theta}_{t}\right) .
$$

In Eq. (11), the terms $V_{n}^{\text {int }}$ and $V^{\text {ext }}$ very much resemble the internal and external potentials obtained from the classical isomorphism in translation path integrals. ${ }^{2}$ The only notable change for the torsional case is the appearance of the integers $n_{j, t}$ in the equation for $V_{n}^{\mathrm{int}}$. This integer term is a manifestation of the constraint that the $\theta_{j, t}$ lies in the interval [0, $2 \pi)$. It accounts for the fact that the distance between two consecutive Trotter beads for a particle $j$, namely $\theta_{j, t}$ and $\theta_{j, t+1}$, may assume not only the explicit value $\theta_{j, t}-\theta_{j, t+1}$ but all of the values $\theta_{j, t}-\theta_{j, t+1}+2 \pi n_{j, t}$. That is, in traveling from one Trotter bead to the next, the chain may wrap around the $[0,2 \pi)$ interval $n_{j, t}$ times. 


\section{B. Winding number representation}

A transformation is now performed to a more compact set of variables. Substituting

$$
\begin{aligned}
& \widetilde{\theta}_{j, 1}=\theta_{j, 1}, \\
& \tilde{\theta}_{j, 2}=\theta_{j, 2}-2 \pi n_{j, 1}, \\
& \widetilde{\theta}_{j, 3}=\theta_{j, 3}-2 \pi n_{j, 1}-2 \pi n_{j, 2}, \\
& \vdots \\
& \widetilde{\theta}_{j, t}=\theta_{j, 2}-2 \pi \sum_{t^{\prime}=1}^{t-1} n_{j, t^{\prime}}
\end{aligned}
$$

leads to a cancellation of all of the $2 \pi n_{j, t}$ terms for $t$ $=1, \ldots, P-1$ :

$$
\left(\theta_{j, t}-\theta_{j, t+1}+2 \pi n_{j, t}\right)^{2} \rightarrow\left(\vec{\theta}_{j, t}-\widetilde{\theta}_{j, t+1}\right)^{2} .
$$

However, the $P$ th term yields

$$
\left(\theta_{j, P}-\theta_{j, P+1}+2 \pi n_{j, P}\right)^{2} \rightarrow\left(\widetilde{\theta}_{j, P}-\widetilde{\theta}_{j, t+P}+2 \pi n_{j}\right)^{2},
$$

where $n_{j}=\Sigma_{t^{\prime}=1}^{P} n_{j, t^{\prime}}$ is known as the winding number. This new set of variables is known as the winding number representation and leads to the following form for $Q:{ }^{15}$

$$
\begin{aligned}
Q(\beta)= & \lim _{P \rightarrow \infty}\left(\frac{P}{2 \pi \beta \hbar^{2}}\right)^{N P / 2} I^{1 / 2} \prod_{j=1}^{N} \sum_{n_{j}=-\infty}^{\infty} \int_{0}^{2 \pi} d \widetilde{\theta}_{j, 1} \\
& \times \prod_{t=2}^{P}\left[\int_{-\infty}^{\infty} d \widetilde{\theta}_{j, t}\right] e^{-\beta\left(V_{n}^{\mathrm{int}}+V^{\mathrm{ext}}\right)}
\end{aligned}
$$

with

$$
V_{\vec{n}}^{\mathrm{int}}=\frac{P}{2 \beta^{2} \hbar^{2}} \sum_{t=1}^{P} \sum_{j=1}^{N} I_{j}\left(\widetilde{\theta}_{j, t}-\widetilde{\theta}_{j, t+1}+2 \pi n_{j} \delta_{t, P}\right)^{2}
$$

and

$$
V^{\mathrm{ext}}=\frac{1}{P} \sum_{t=1}^{P} V\left(\overrightarrow{\widetilde{\theta}}_{t}\right) .
$$

The periodicity of the potential renders the expression for $V^{\text {ext }}$ unchanged from Eq. (11). The winding number $n_{j}$ in this representation is a property of the entire ring of Trotter beads for a given particle $j$. It represents the net number of times that the ring loops around the interval $[0,2 \pi)$. Introduction of the winding number in this fashion frees all particles from the $[0,2 \pi)$ boundary constraint, except for particle $j=1$. The winding number representation for rotational path integrals has been utilized extensively by Marx and co-workers in the study of dimers rotating on a surface. ${ }^{14,15}$

\section{Uncoupled winding number representation}

Another change of variables is still necessary before we arrive at the path integral formulation most readily applicable to molecular torsions. The following variable substitution was first suggested by Cao. ${ }^{23}$

$$
\bar{\theta}_{j, t}=\widetilde{\theta}_{j, t}+2 \pi n_{j}\left(\frac{t-1}{P}\right) t=1, \ldots, P .
$$

Transformation to the uncoupled winding number representation leaves the following form of the partition function:

$$
\begin{aligned}
Q(\beta)= & \lim _{P \rightarrow \infty}\left(\frac{P}{2 \pi \beta \hbar^{2}}\right)^{N P / 2} I^{1 / 2} \prod_{j=1}^{N} \int_{0}^{2 \pi} d \bar{\theta}_{j, 1} \\
& \times \prod_{t=2}^{P}\left[\int_{-\infty}^{\infty} d \bar{\theta}_{j, t}\right] \sum_{n_{j}=-\infty}^{\infty} e^{-\beta\left(V^{\mathrm{int}}+V_{\bar{n}}^{\mathrm{ext}}+V_{n}^{\text {wind }}\right)} .
\end{aligned}
$$

The terms $V^{\text {int }}, V_{\vec{n}}^{\text {ext }}$, and $V_{\vec{n}}^{\text {wind }}$ assume the form

$$
\begin{aligned}
& V^{\mathrm{int}}=\frac{P}{2 \beta^{2} \hbar^{2}} \sum_{j=1}^{N} I_{j}\left[\sum_{t=1}^{P}\left(\overrightarrow{\bar{\theta}}_{j, t}-\bar{\theta}_{j, t+1}\right)^{2}\right], \\
& V_{\vec{n}}^{\mathrm{ext}}=\frac{1}{P} \sum_{t=1}^{P} V\left(\theta_{t}-\frac{2 \pi \vec{n}(t-1)}{P}\right),
\end{aligned}
$$

and

$$
V_{\vec{n}}^{\text {wind }}=\frac{1}{2 \beta^{2} \hbar^{2}} \sum_{j=1}^{N} I_{j}\left(2 \pi n_{j}\right)^{2} .
$$

The uncoupled winding number representation is a useful formulation of torsional PIMC theory, largely because of its strong similarities to the formalism of translation PIMC. The expressions for $V^{\text {int }}$ and $V_{n}^{\text {ext }}$ are virtually unchanged from their form for the case of translation PIMC: ${ }^{38}$

$$
V_{\text {int }}=\frac{m P}{2 \beta^{2} \hbar^{2}} \sum_{n=1}^{P}\left(x_{n}-x_{n+1}\right)^{2}
$$

and

$$
V_{\mathrm{ext}}=\frac{1}{P} \sum_{n=1}^{P} V\left(x_{n}\right)
$$

Also, the winding number terms $n_{j}$ in Eq. (18) are, aside from the coordinate shift in $V_{n}^{\text {ext }}$, decoupled from the angular coordinates. Computationally, the expression for $Q$ in Eq. (18) is implemented by choosing maximum values of $P$ and $n_{j}$. Suitable maximum values of the $n_{j}$ are apparent from the uncoupled expression because the entire winding number contribution for a given particle falls away exponentially with $I_{j}$ and $n_{j}^{2}$.

Equation (18) suggests that a Monte Carlo sampling distribution of $\rho=(1 / Q) e^{-\beta\left(V^{\mathrm{int}}+V_{0}^{\mathrm{ext}}\right)}$ is appropriate..$^{23}$ The path integral (PI) energy estimator is then

$$
E^{\mathrm{PI}}=\prod_{j=1}^{N}\left[\sum_{n_{j}=-n_{j}^{\max }}^{n_{j}^{\max }}\right] \epsilon_{\vec{n}}^{\mathrm{PI}} e^{-\Delta S_{\vec{n}}},
$$

where $n_{j}^{\max }$ is the maximum winding number for a given particle $j$;

$$
\begin{aligned}
\Delta S_{\vec{n}}= & \beta\left(\frac{1}{P} \sum_{t=1}^{P}\left[V\left(\overrightarrow{\bar{\theta}}_{t}-\frac{2 \pi \vec{n}(t-1)}{P}\right)-V\left(\overrightarrow{\bar{\theta}}_{t}\right)\right]\right. \\
& \left.+\frac{1}{2 \beta^{2} \hbar^{2}} \sum_{j=1}^{N} I_{j}\left(2 \pi n_{j}\right)^{2}\right),
\end{aligned}
$$

and

$$
\epsilon_{\vec{n}}^{\mathrm{PI}}=\frac{N P}{2 \beta}-V^{\mathrm{int}}+V_{\vec{n}}^{\mathrm{ext}}-V_{\vec{n}}^{\mathrm{wind}} .
$$


The equations for $V^{\text {int }}, V_{n}^{\text {ext }}$, and $V_{\vec{n}}^{\text {wind }}$ were provided in Eq. (19). The weight $\omega$ of each configuration is also calculated using

$$
\omega=\prod_{j=1}^{N}\left[\sum_{n_{j}=-n_{j}^{\max }}^{n_{j}^{\max }}\right] e^{-\Delta S_{\vec{n}}}
$$

to yield the average internal energy expectation value

$$
\langle E\rangle=\frac{\sum_{\text {configs }} E^{\mathrm{PI}}}{\sum_{\text {configs }} \omega} .
$$

The path integral energy estimator presented here is often referred to as the kinetic energy estimator. ${ }^{1}$ Another popular path integral energy estimator is the virial energy estimator, which is often found to give better numerical convergence for path integral simulations requiring a large number of Trotter beads. ${ }^{38}$ However, it is not found that the coupled molecular torsion problem requires large numbers of Trotter beads, so since the virial energy estimator requires the computationally expensive task of determining energy gradients, we discuss only the kinetic energy estimator in this study.

The scaling of the method is dominated by the term:

$$
\prod_{j=1}^{N}\left[2 n_{j}^{\max }+1\right] P N^{\mathrm{MC}},
$$

where $N^{\mathrm{MC}}$ is the the number of steps in the Monte Carlo simulation. Consideration of only torsional degrees of freedom in the current path integral formulation yields a significant reduction in computational cost. For systems in which vibrational degrees of freedom are not expected to contribute significantly to calculated observables, the torsional PIMC technique will serve as a most effective alternative to conventional PIMC algorithms.

It is easily verified that a classical simulation may be performed within the PIMC formalism by simply setting the number of Trotter beads $P$ to 1 .

\section{Simplification of winding number term}

As was noted in Sec. II C, an advantage of the uncoupled winding number representation is the easily determined influence of winding numbers in the evaluation of expectation values for a given system. Equation (21) shows that contributions to the internal energy from a given torsion in a given configuration fall away exponentially with the term

$$
\beta V_{j}^{\text {wind }}=\frac{1}{2 \beta \hbar^{2}} I_{j}\left(2 \pi n_{j}\right)^{2} .
$$

However, for even the lightest molecular torsions, the term $I_{j}$ will generally exceed $10^{3}$ a.u. Thus, at standard temperature,

$$
\begin{aligned}
\beta V_{j}^{\text {wind }} & =\frac{1}{2 \beta \hbar^{2}} I_{j}\left(2 \pi n_{j}\right)^{2} \\
& \sim \frac{1}{2(1156)(1)^{2}}(1000)\left(2 \pi n_{j}\right)^{2} \\
& \sim 17 n_{j}^{2} .
\end{aligned}
$$

Clearly, the only exponential terms small enough to contribute to the expectation value will thus correspond to $n_{j}=0$, so we can reliably set $n_{\max }=0$ for molecular calculations. This dramatically improves the scaling of the method, as is evident from Eq. (26).

\section{CALCULATION DETAILS}

The molecular systems considered in this study were ethane, $n$-butane, $n$-octane, and the peptide enkephalin. These molecules include, respectively, 1, 3, 7, and 33 internal torsions.

Two potentials are employed in this study. The first is an analytical potential used only for the ethane molecule. The analytical ethane potential is a one-dimensional (1D) sinusoidal function of the torsional angle $\theta$. Parametrizing the maxima of the potential to the MM3Pro force field yielded the function $V(\theta)=C(\cos (3 \theta)+1)$, where $C$ $=1.33 \mathrm{kcal} \mathrm{mol}^{-1} \cdot{ }^{39}$ The second potential was used for all of the molecules studied. This latter potential is the MM3Pro atom-atom force field as implemented in the TINKER molecular mechanics package. ${ }^{39,40}$

The TPIMC code written for the purposes of this study evaluates the internal energy of an arbitrary molecule. Subroutines from the TINKER molecular mechanics package were included to perform the changes between Cartesian and internal coordinates and to evaluate the energy of the system using a molecular mechanics force field. ${ }^{40}$ Also, the MINIMIZE program in the TINKER package was used to optimize the geometry of each molecule before performing the internal energy calculations. ${ }^{41}$ In no case was a lower energy structure found during the course of the Monte Carlo simulation. Calculations are performed using a variety of Trotter beads ranging from 1 to 15 , and the impact of this number is evaluated. The exponentially decaying influence discussed in Sec. II D of winding number terms other than the nonzero term, along with preliminary calculations which included these terms, showed that the maximum winding number parameter $n_{\max }$ could be set to 0 . The Monte Carlo selection process was performed with the assistance of a simple Metropolis algorithm, and the changes in bead coordinates are performed according to a random walk algorithm. ${ }^{42}$ During the Monte Carlo simulation, two types of random geometry steps are performed. The first type allows each Trotter bead to move with respect to the other beads in the corresponding ring. The second type of step moves an entire ring of Trotter beads as a unit. For each ring, parameters limiting the maximum size of each type of random step are defined. For each molecule, 20 TPIMC simulations, each consisting of $10^{5}$ Monte Carlo steps, are performed. Energy samples are performed once every 20 Monte Carlo steps to reduce correlation of the sampled values. All TPIMC calculations are initiated at the minimum-energy geometry of the molecule, but the energy samples obtained from the first 500 Monte Carlo steps are discarded to eliminate the impact of this starting point. The maximum step size parameters are updated every 50 steps during these discarded cycles to yield an acceptance ratio of 0.5 . For each molecule, the torsional moments of inertia are calculated only once at the beginning of the calculation. In no case are large-scale geometric changes ob- 
served, so the moments of inertia are assumed to remain effectively unchanged during the course of the simulation. The computation time required for the TPIMC algorithm on a $800 \mathrm{MHz}$ Pentium III processor is approximately $20 \mathrm{~h} / \mathrm{bead}$ for the enkephalin peptide, the largest system studied.

In addition to the TPIMC technique, we employ a harmonic oscillator approximation to the torsional angle and a variational technique. The harmonic oscillator approximation may be used to determine the internal energy analytically for a system of $N$ uncoupled torsions using the expression

$$
\langle E\rangle=\sum_{j=1}^{N} \frac{\hbar \omega_{j}}{2}+\sum_{j=1}^{N} \frac{\hbar \omega_{j} e^{-\beta \hbar \omega}}{1-e^{-\beta \hbar \omega}},
$$

where $\omega_{j}=\sqrt{k_{j} / I_{j}}$, and $k_{j}$ and $I_{j}$ are, respectively, the force constant and moment of inertia of the $j$ th torsion. Note that this formulation of the harmonic oscillator approximation neglects both the anharmonicity of the molecular torsions and the coupling between the individual oscillators. The harmonic oscillator and TPIMC results are compared for all systems and potentials used in this study.

A variational technique was used only for the simple ethane model with the analytical potential. Using a large basis set of free-particle wave functions subjected to periodic boundary constraints, the eigenfunctions and eigenvalues of the model ethane system were obtained to high numerical accuracy.

\section{RESULTS AND DISCUSSION}

The internal energies of the molecules ethane, $n$-butane, $n$-octane, and enkephalin were determined using the methods described in Sec. III. Whereas the TPIMC technique and the harmonic oscillator approximation were utilized for each molecule, the exact variational technique was employed only for the ethane molecule.

\section{A. Simple ethane model}

The ethane molecule serves as a simple, yet realistic system with which the various methods can be compared. In this study, the TPIMC technique, the variational technique, and the harmonic oscillator approximation were all employed for the simple ethane model. The analytical potential energy surface used for ethane is described in Sec. III. A comparison of the PIMC technique and a quasiharmonic technique for crystalline polyethylene has been previously reported. ${ }^{20,21}$ However, the torsional path integral formulation employed in this study is computationally less expensive and will ultimately enable the study of larger molecular systems.

Figure 1 displays the numerical results for the internal energy at $273.15 \mathrm{~K}$ obtained with the various methods. For the analytical potential, the variational result was easily obtained to extremely high accuracy, and the reported variational value of $0.870 \mathrm{kcal} \mathrm{mol}^{-1}$ is presumed to be exact for the model. The TPIMC algorithm was performed using one, two, three, four, five, and ten Trotter beads. The TPIMC method performed with one Trotter bead yields the classical statistical mechanics result. The one-bead TPIMC result of $0.585 \mathrm{kcal} \mathrm{mol}^{-1}$ is accordingly labeled in the figure.

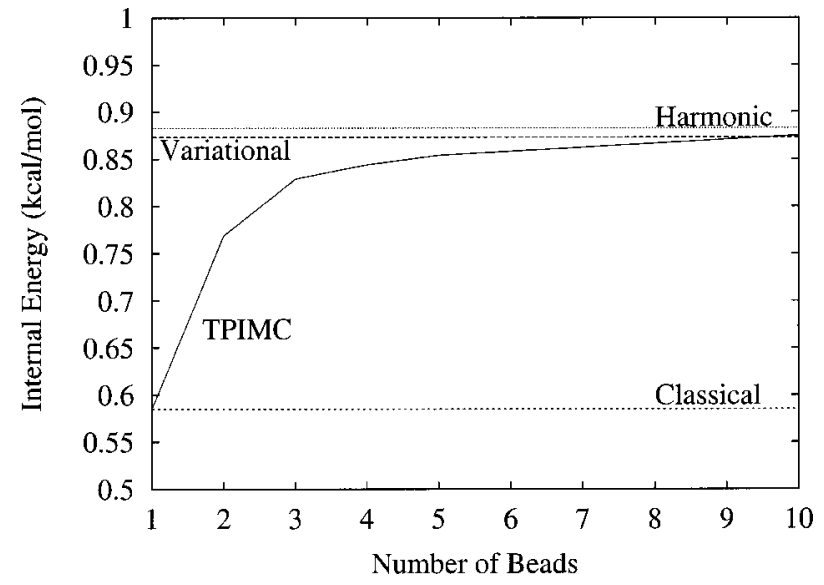

FIG. 1. Calculated internal energy for ethane model at $273.15 \mathrm{~K}$.

Also included in Fig. 1 is the value $0.883 \mathrm{kcal} \mathrm{mol}^{-1}$ of the internal energy predicted with the harmonic oscillator approximation.

Two important features of Fig. 1 require discussion. The first is that, as required, the internal energy calculated using the TPIMC algorithm converged with the increase of Trotter beads to the exact variational result. Whereas the majority of the quantum contribution to the internal energy is recovered upon inclusion of a second Trotter bead, greater than five beads is required for this simple ethane model to recover the exact result. Using ten Trotter beads, the TPIMC method has converged to within numerical accuracy for the simple problem.

Of the entire alkane series ethane is, in a sense, the most quantum mechanical. It is a standard result from the theory of quantum mechanical tunneling that the wave function of a particle in a classically forbidden region diminishes exponentially with the square root of the mass of the particle. ${ }^{43}$ Translating this observation to the current application, we note that the degree of quantum behavior of torsions with large moments of inertia is expected to be drastically smaller than that of torsions with small moments of inertia. Suppose that $I_{1}$ and $I_{2}$ are the moments of inertia of two substituent groups about the bond that connects them. The moment of inertia about this torsion is then $I_{1} I_{2} /\left(I_{1}+I_{2}\right)$. Presume, momentarily, that only the terminal methyl groups are small enough in an unbranched alkane to exhibit quantum behavior. Define $I_{m}$ to be the moment of inertia for a methyl substituent, and define $I_{x}$ to be the considerably larger moment of inertia for the rest of the molecule. Therefore, for all unbranched alkanes longer than ethane, the molecule will exhibit two torsions with moment of inertia

$$
I_{\mathrm{tor}}=\frac{I_{m} I_{x}}{I_{m}+I_{x}} \sim \frac{I_{m} I_{x}}{I_{x}}=I_{m} .
$$

On the other hand, the lone torsion in ethane will exhibit a moment of inertia equal to that of $I_{m} / 2$. This single torsion in the ethane molecule is expected to contribute more to the quantum mechanical portion of the internal energy than the combined contributions of the two methyl torsions in a longer alkane chain. 


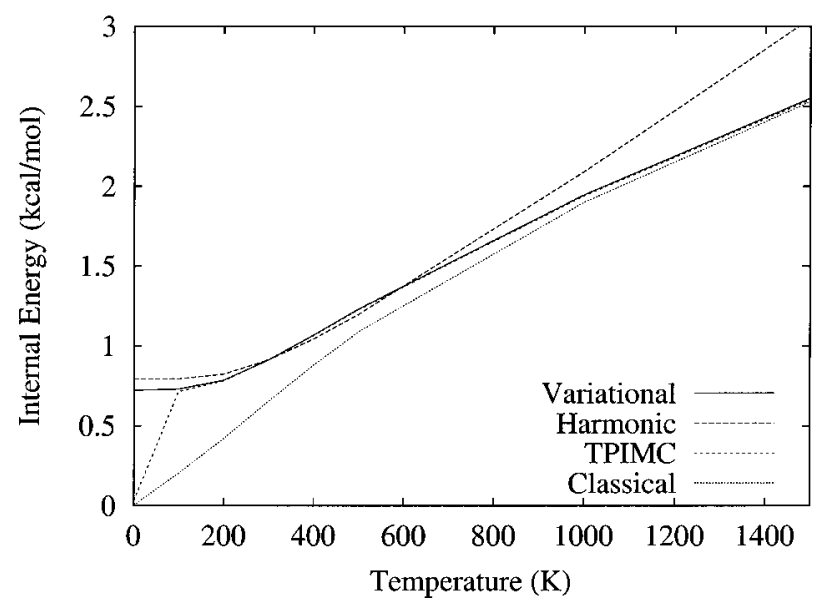

FIG. 2. Internal energy for ethane model calculated at various temperatures.

Of course, the above analysis of moments of inertia is approximate, but it does lend credence to the likelihood that larger molecules will not require as many Trotter beads as the simple ethane model required for the TPIMC value to converge to the exact quantum mechanical result. For larger systems, the TPIMC value of internal energy is reported for the same array of Trotter beads, and we note the number of beads necessary to observe the plateau of convergence.

A second important feature of Fig. 1 is that the harmonic oscillator performs very well in comparison to the exact variational result. As one might have predicted from a comparison of the harmonic and sinusoidal potentials, the steeper walls of the harmonic potential drive the calculated internal energy above the exact variational result. However, the relationship between the harmonic approximation and variational internal energies is more complicated than this result suggests. In Fig. 2, the calculated internal energies for the simple ethane model are presented as a function of temperature. In this figure, the TPIMC results are obtained with 15 Trotter beads, and the classical results are obtained from the TPIMC technique by utilizing a single Trotter bead.

For both the low and high temperature extremes, the harmonic approximation behaves as expected. In the low temperature extreme, only the ground state of the system is occupied. The steeper curvature of the harmonic potential increases the approximated ground state energy with respect to the exact result. Figure 2 shows that the harmonic approximation internal energy of $0.7928 \mathrm{kcal} \mathrm{mol}^{-1}$ overestimates the exact variational result of $0.7212 \mathrm{kcal} \mathrm{mol}^{-1}$ as $T \rightarrow 0$. Note that, as predicted by the Trotter product formula in Eq. (4), the TPIMC and classical results break down in this low temperature extreme.

In the high temperature limit, the harmonic and variational results naturally diverge as the harmonic approximation of the sinusoidal potential deteriorates. With increasing quantum number, the exact wave functions of the sinusoidal potential converge to those of the free particle, not the harmonic oscillator. Increased thermal population of the high energy oscillator states in the harmonic approximation drive the approximated internal energy steadily higher than the exact result. Both the TPIMC and classical results correctly converge to the exact result in the high temperature extreme

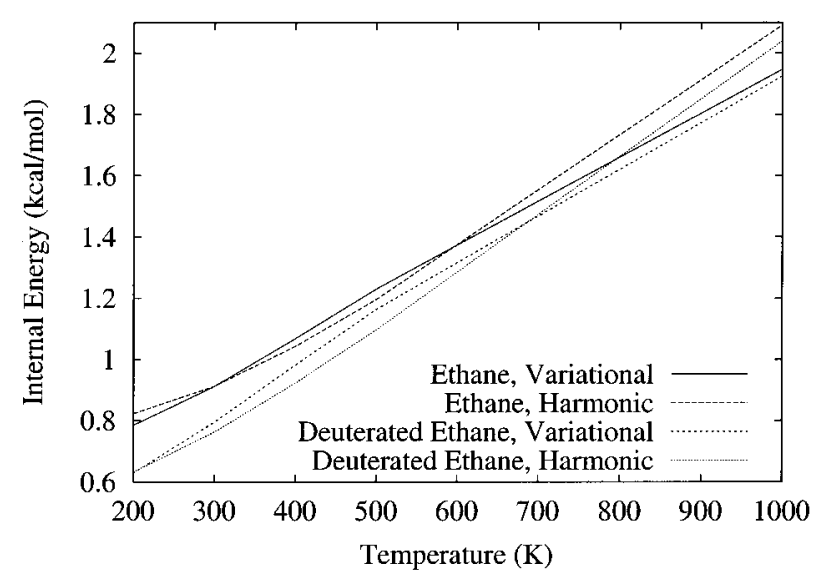

FIG. 3. Internal energies for ethane and deuterated ethane models calculated at various temperatures.

as the error term in the Trotter product formula goes to zero. As seen in Fig. 2, the variational, TPIMC, and classical internal energies all approach the value of $2.553 \mathrm{kcal} \mathrm{mol}^{-1}$ at $1500 \mathrm{~K}$.

There exists in Fig. 2 an intermediate temperature range for which the internal energy calculated with the harmonic approximation falls below the exact result. This temperature range is expanded in Fig. 3. Also presented in Fig. 3 are the harmonic approximation and exact variational results for the ethane model in which all the hydrogens in the molecule has been replaced with deuterium atoms.

For the simple ethane model, the harmonic approximation falls below the exact internal energy between 300 and $600 \mathrm{~K}$. Although the energies of the harmonic oscillator states are regularly spaced, such is not the case for the eigenfunctions of the sinusoidal potential. These exact eigenfunctions become more dense with increasing energy. Thus, there may exist a temperature range for which the increased number of energetically accessible quantum states in the sinusoidal potential raises the exact variational internal energy in excess of the harmonic approximation internal energy. Interestingly, this effect becomes more pronounced with increasing moments of inertia. It may be seen in Fig. 3 that the temperature range for which the deuterated ethane exact and harmonic internal energies are inverted is extended to between 200 and $700 \mathrm{~K}$. Figure 3 suggests that the harmonic approximation will become increasingly weak for larger moments of inertia.

It is worthwhile to note that in Fig. 2, the TPIMC results determined with 15 Trotter beads accurately reproduce the exact internal energies for all temperatures except those in the low-energy extreme. Performance of the TPIMC technique at low temperatures can be improved by simply increasing the number of Trotter beads.

\section{B. Larger molecules}

In this section, we report the TPIMC and harmonic approximation results for the four molecules: ethane, $n$-butane, $n$-octane, and enkephalin. The unbranched alkanes ethane, 
TABLE I. Calculated moments of inertia of ethane, $n$-butane, and $n$-octane in a.u. $\times 10^{3}$.

\begin{tabular}{cccc}
\hline \hline & Ethane & $n$-butane & $n$-octane \\
\hline 1 & 2.99 & 5.70 & 5.94 \\
2 & $\cdots$ & 34.8 & 65.3 \\
3 & $\cdots$ & 5.70 & 104 \\
4 & $\cdots$ & $\cdots$ & 177 \\
5 & $\cdots$ & $\cdots$ & 104 \\
6 & $\cdots$ & $\cdots$ & 65.3 \\
7 & $\cdots$ & $\cdots$ & 5.94 \\
\hline \hline
\end{tabular}

$n$-butane, and $n$-octane have, respectively, one, three, and seven torsions. The calculated values of these moments of inertia are reported in Table I.

Enkephalin is a considerably larger molecule than the three considered alkanes. Displayed in Fig. 4, it contains 75 atoms comprising 33 torsional degrees of freedom. However, only three of these torsions have moments of inertia less than $10^{4}$ a.u. This feature portends smaller quantum effects for enkephalin than would be expected for more heavily branched molecules exhibiting a greater number of terminal methyl groups.

The internal energies of ethane, $n$-butane, $n$-octane, and enkephalin calculated with the TPIMC method are reported in Table II. For the three alkanes, TPIMC calculations were performed with one, two, three, four, five, and ten Trotter beads. The enkephalin internal energies were determined with only one, two, three, four, and five Trotter beads.

First of all, it is important to compare the results obtained for ethane with the MM3Pro potential implemented in

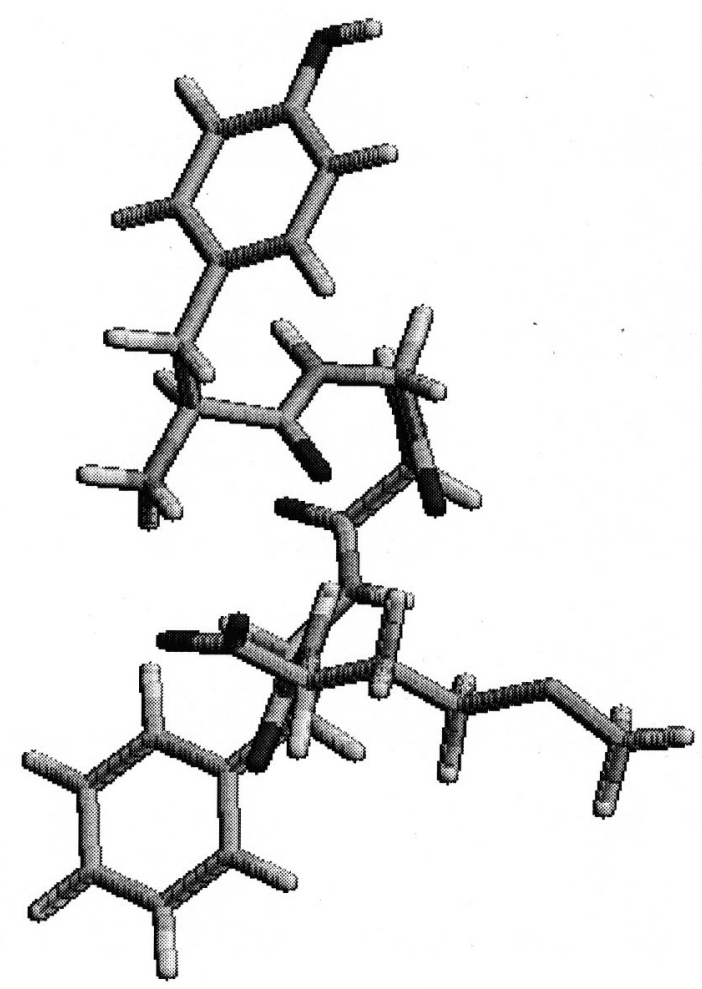

FIG. 4. Structure of the enkephalin molecule.

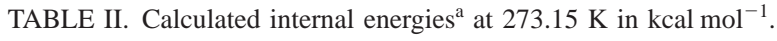

\begin{tabular}{rcccc}
\hline \hline$P$ & Ethane & $n$-butane & $n$-octane & Enkephalin \\
\hline 1 & $0.594(1)$ & $1.97(2)$ & $4.99(5)$ & $17.9(2)$ \\
2 & $0.758(1)$ & $2.27(2)$ & $5.26(8)$ & $19.1(1)$ \\
3 & $0.810(1)$ & $2.33(2)$ & $5.32(8)$ & $19.0(3)$ \\
4 & $0.830(2)$ & $2.32(2)$ & $5.34(7)$ & $19.4(1)$ \\
5 & $0.841(2)$ & $2.37(2)$ & $5.35(7)$ & $19.5(1)$ \\
10 & $0.855(4)$ & $2.43(2)$ & $5.34(8)$ & \\
\hline \hline
\end{tabular}

${ }^{a}$ Numbers in parentheses indicate standard deviation in last significant digit.

${ }^{\mathrm{b}}$ The notation here indicates a standard deviation of $1.4 \mathrm{kcal} \mathrm{mol}^{-1}$.

TINKER, as opposed to the sinusoidal potential parametrized to MM3Pro discussed in the previous section. For the true MM3Pro potential, the TPIMC method predicts classical and quantum energies of 0.594 and $0.86 \mathrm{kcal} \mathrm{mol}^{-1}$, respectively, which agree well with the values of 0.585 and 0.875 $\mathrm{kcal} \mathrm{mol}^{-1}$ reported for the parametrized analytical potential. This suggests correct implementation of the TPIMC code for general molecular systems.

For each model, we note the number of beads required before the calculated internal energy remains constant (within numerical accuracy) with respect to an increase in the number of beads. As observed for the model ethane system, at least five Trotter beads are required for the ethane internal energy to reach convergence. The other molecules, however, are more classical in behavior. Neither $n$-butane, $n$-octane, nor enkephalin exhibit numerically significant changes in internal energy beyond the third Trotter bead. That is, a calculation with one Trotter bead is performed to obtain the classical internal energy, and another calculation with only two or three Trotter beads is needed to obtain the quantum internal energy.

Despite the small number of required Trotter beads, there is a substantial quantum contribution to the internal energy for every molecule studied. The quantum mechanical contributions to the internal energy of ethane and $n$-butane are, respectively, seen to comprise $31 \%$ and $19 \%$ of the total calculated internal energies. The significance of this proportion diminishes rapidly with the size of the molecules, however, and both $n$-octane and enkephalin are seen to have quantum contributions of only $\sim 7 \%$. Nonetheless, the 1 $\mathrm{kcal} \mathrm{mol}^{-1}$ of quantum internal energy in enkephalin is far from negligible and strongly suggests the need for consideration of quantum mechanical behavior in large biomolecules.

Another comparison of the TPIMC technique and the harmonic oscillator approximation is possible with the data presented in Table III, which includes the TPIMC results for ethane, $n$-butane, and $n$-octane with ten Trotter beads and the TPIMC result for enkephalin with five Trotter beads. Also displayed are the results obtained with the harmonic oscillator approximation.

Similarly to the results reported in Fig. 1 for the simple ethane model, the harmonic oscillator results in Table III perform strongly in comparison to the TPIMC results. Nonetheless, as the size of the molecules increase, the larger moments of inertia lead the harmonic approximation to underestimate the actual internal energy (see Fig. 3). Although the impact of the deviation of the harmonic approximation is still 
TABLE III. TPIMC and harmonic oscillator approximation internal energies for ethane, $n$-butane, $n$-octane, and enkephalin at $273.15 \mathrm{~K} \mathrm{in} \mathrm{kcal} \mathrm{mol}^{-1}$.

\begin{tabular}{lcccc}
\hline \hline & Ethane & $n$-butane & $n$-octane & Enkephalin \\
\hline TPIMC & 0.86 & 2.43 & 5.3 & 19.5 \\
Harmonicoscillation & 0.86 & 2.08 & 4.27 & 19.14 \\
\hline \hline
\end{tabular}

relatively small for the molecules presented in Table III, the systematic error could become more significant for larger and more anharmonic molecules.

\section{CONCLUSIONS}

In this study, we apply the uncoupled winding number formulation of path integral Monte Carlo theory to the torsional degrees of freedom in the molecules ethane, $n$-butane, $n$-octane, and enkephalin. This TPIMC technique offers a significant reduction in computational cost for systems in which vibrational degrees of freedom may be safely neglected. Employment of the TPIMC technique is simplified by the observation that contributions to calculated properties will be negligible for winding numbers greater than zero. For a simple ethane model potential, the TPIMC result recovers the exact internal energy value obtained with a variational technique. For $n$-butane, $n$-octane, and enkephalin at standard temperature, the TPIMC converged to the quantum mechanical limit with only two Trotter beads. All molecules studied exhibited significant quantum mechanical contributions to their internal energies at standard temperature according to the TPIMC technique.

Although the harmonic oscillator approximation method for calculating the internal energy performs well in comparison to the TPIMC technique for the molecules presented in this study, there exists a tendency in larger molecules for the harmonic approximation to underestimate the results obtained with the more rigorous TPIMC technique. It was illustrated with the simple ethane model that this underestimation is related to the size of the molecular moments of inertia, suggesting a systematic shortcoming in the application of the harmonic oscillator approximation to larger systems. Moreover, the harmonic approximation includes neither the coupling between torsions nor the effects of anharmonicity intrinsically accounted for with the TPIMC technique. These effects are likely to be more important in larger molecules, such as proteins, which are currently under investigation.

\section{ACKNOWLEDGMENTS}

The authors would like to thank Dr. Jianshu Cao and Dr. Seogjoo Jang for their helpful discussions regarding the uncoupled winding number representation of rotational path integrals. T.F.M. gratefully acknowledges financial assistance from the Marshall Aid Commemoration Commission. D.C.C. acknowledges a research fellowship from the Leverhulme Trust. The work was supported by the Engineering and Physical Sciences Research Council.

${ }^{1}$ J. A. Barker, J. Chem. Phys. 70, 2914 (1979).

${ }^{2}$ D. Chandler and P. G. Wolynes, J. Chem. Phys. 74, 4078 (1981).

${ }^{3}$ B. J. Berne and D. Thirumalai, Annu. Rev. Phys. Chem. 37, 401 (1986).

${ }^{4}$ B. J. Berne and D. Thirumalai, Quantum Simulations of Condensed Matter Phenomena (World Scientific, Singapore, 1990).

${ }^{5}$ D. Chandler, Liquids, Freezing and Glass Transition (Elsevier, Amsterdam, 1991).

${ }^{6}$ J. D. Doll and D. L. Freeman, J. Chem. Phys. 80, 2239 (1980).

${ }^{7}$ D. Chandler, J. Phys. Chem. 88, 3400 (1984).

${ }^{8}$ J. D. Doll, R. D. Coalson, and D. L. Freeman, Phys. Rev. Lett. 55, 1 (1986).

${ }^{9}$ J. D. Doll and L. E. Meyers, J. Chem. Phys. 71, 2880 (1979).

${ }^{10}$ K. J. Runge, M. P. Surh, C. Mailhiot, and E. L. Pollock, Phys. Rev. Lett. 69, 3527 (1992).

${ }^{11}$ Y. H. Lee and B. J. Berne, J. Phys. Chem. A 104, 86 (2000).

${ }^{12}$ Y. H. Lee and B. J. Berne, J. Phys. Chem. A 105, 459 (2001).

${ }^{13}$ T. G. Dewey, Phys. Rev. E 60, 4652 (1999).

${ }^{14}$ D. Marx and M. H. Muser, J. Phys.: Condens. Matter 11, R117 (1999).

${ }^{15}$ D. Marx and P. Nielaba, Phys. Rev. A 45, 8968 (1992).

${ }^{16}$ K. J. Runge, M. P. Surh, C. Mailhiot, and E. L. Pollock, Phys. Rev. Lett. 69, 3527 (1992).

${ }^{17}$ S. L. Mielke, J. Srinivasan, and D. G. Truhlar, J. Chem. Phys. 112, 8758 (2000).

${ }^{18}$ S. L. Mielke and D. G. Truhlar, J. Chem. Phys. 114, 621 (2001).

${ }^{19}$ S. L. Mielke and D. G. Truhlar, J. Chem. Phys. 115, 652 (2001).

${ }^{20}$ G. C. Rutledge, D. J. Lacks, R. Martoňák, and K. Binder, J. Chem. Phys. 108, 10274 (1998).

${ }^{21}$ R. Martoňák, W. Paul, and K. Binder, Phys. Rev. E 57, 2425 (1998).

${ }^{22}$ D. C. Clary, J. Chem. Phys. 114, 9725 (2001).

${ }^{23}$ J. Cao, Phys. Rev. E 49, 882 (1994).

${ }^{24}$ R. P. Feynman and A. R. Hibbs, Quantum Mechanics and Path Integrals (McGraw-Hill, New York, 1965).

${ }^{25}$ R. P. Feynman, Statistical Mechanics (Benjamin, Reading, MA, 1972).

${ }^{26}$ L. S. Schulman, Techniques and Applications of Path Integrations (Wiley, New York, 1981).

${ }^{27}$ M. H. Kalos, Phys. Rev. A 2, 250 (1970).

${ }^{28}$ M. H. Kalos, D. Levesque, and L. Verlet, Phys. Rev. A 9, 2178 (1974).

${ }^{29}$ P. A. Whitlock and M. H. Kalos, J. Comput. Phys. 30, 361 (1979).

${ }^{30}$ D. M. Ceperley and M. H. Kalos, Monte Carlo Methods in Statistical Physics (Springer, Berlin, 1981).

${ }^{31}$ M. Suzuki, Commun. Math. Phys. 56, 183 (1976).

${ }^{32}$ M. Suzuki, Quantum Monte Carlo Methods in Equilibrium and Nonequilibrium Systems (Springer, Berlin, 1987).

${ }^{33}$ H. F. Trotter, Proc. Am. Math. Soc. 10, 545 (1959).

${ }^{34} \mathrm{H}$. Kleinert, Path Integrals in Quantum Mechanics, Statistics and Polymer Physics (World Scientific, Singapore, 1990).

${ }^{35}$ A. Vivier-Bunge, V. H. Uc, and Y. G. Smeyers, J. Chem. Phys. 109, 2279 (1998).

${ }^{36} \mathrm{R}$. Bellman, A Brief Introduction to Theta Functions (Holt, Rinehart, and Winston, New York, 1961).

${ }^{37}$ W. K. Burton and A. H. De Borde, Nuevo Cimento 2, 197 (1955).

${ }^{38}$ M. F. Herman, E. J. Bruskin, and B. J. Berne, J. Chem. Phys. 76, 5150 (1982).

${ }^{39}$ N. L. Allinger, Y. H. Yuh, and J. H. Li, J. Am. Chem. Soc. 111, 8551 (1989).

${ }^{40}$ J. W. Ponder, TINKER Software Tools for Molecular Design, Version 3.8 (2000).

${ }^{41}$ J. Nocedal, Math. Comput. 35, 773 (1980).

${ }^{42}$ N. Metropolis, A. W. Rosenbluth, M. N. Rosenbluth, A. H. Teller, and E. Teller, J. Chem. Phys. 21, 1087 (1953).

${ }^{43}$ J. J. Sakurai, Modern Quantum Mechanics (Addison-Wesley, New York, 1994). 